\title{
Adaptive Sensitivity Analysis with Nonlinear Power Load Modeling
}

\author{
Po-Ya Hsu, Chun-Han Yao, Yuwei Wang, and Chung-Kuan Cheng \\ CSE Dept., University of California, San Diego \\ La Jolla, California \\ p8hsu@ucsd.edu,chy235@ucsd.edu,yuw267@ucsd.edu,ckcheng@ucsd.edu
}

\begin{abstract}
Voltage fluctuation in power networks is a critical issue for VLSI designs. The analysis and optimization of the voltage drops rely on accurate sensitivity calculation. Due to the high complexity of largescale circuits, in practice active devices are simplified with power load models. In order to grasp the details of voltage fluctuation, we propose a nonlinear power load model as a function of the supply voltage. We adopt an adaptive analysis algorithm to accelerate the calculation. The power load model provides efficient and accurate noise prediction at system-level.
\end{abstract}

\section{KEYWORDS}

power load model, sensitivity analysis, noise prediction, systemlevel

ACM Reference Format:

Po-Ya Hsu, Chun-Han Yao, Yuwei Wang, and Chung-Kuan Cheng. 2018. Adaptive Sensitivity Analysis with Nonlinear Power Load Modeling. In SLIP '18: SLIP '18: System Level Interconnect Prediction Workshop, fune 23, 2018, San Francisco, CA, USA. ACM, New York, NY, USA, 6 pages. https: //doi.org/10.1145/3225209.3225211

\section{INTRODUCTION}

Due to the ever shrinking size of VLSI design, the effect of voltage fluctuation in power distribution network (PDN) has become increasingly significant. As shown in the ITRS roadmap [4], the current density of a single chip keeps increasing while the operating voltage of high performance processors is gradually dropping, which makes the target impedance of a PDN lower than ever. Meanwhile, Kim et al. [6] observed that the full-chip leakage power has increased by three times in recent years. Consequently, we can no longer neglect the IR drop and simultaneous switching noise (SSN) caused by parasitic capacitances and the short circuit currents that occur when NMOS and PMOS devices are switching.

With the increasing need of accurate PDN analysis, several works have been proposed for impedance modeling and noise minimization. Zhang et al. [12] minimized power supply noise in 3D IC using power transmission line based design. Charles et al. [1] studied

Permission to make digital or hard copies of all or part of this work for personal or classroom use is granted without fee provided that copies are not made or distributed for profit or commercial advantage and that copies bear this notice and the full citation on the first page. Copyrights for components of this work owned by others than ACM must be honored. Abstracting with credit is permitted. To copy otherwise, or republish to post on servers or to redistribute to lists, requires prior specific permission and/or a fee. Request permissions from permissions@acm.org.

SLIP '18, June 23, 2018, San Francisco, CA, USA

(C) 2018 Association for Computing Machinery.

ACM ISBN 978-1-4503-5900-9/18/06 . \$ \$15.00

https://doi.org/10.1145/3225209.3225211
PDN effect of four chip-stacking topologies. Tanaka et al. [11] measured the PDN impedance profile and SSN of 3D system in a single package. Smith [10] proposed to characterize on-die PDN noise and generate the worst-case current stimuli. Kim [7] estimated the supply noise from the frequency-domain PDN impedance profile. Kim et al. [5] gave the closed-form expressions of the power supply noise caused by IC switching current for a complete PDN structure.

In previous works, the model of short circuit current is either ignored [7] [5] or considered as a constant [10] [8] in system-level PDN design. Zhang et al. [13] modeled the resistance as a linear function of supply voltage. Although it is demonstrated to predict PDN noise accurately and efficiently, the high performance is limited to the sub-threshold region of an NMOS.

In this work we focus on voltage-dependent power load modeling to accelerate large-scale simulation while maintaining high accuracy. Specifically, power loads are represented by a combination of Resistance and Current Source (RI) activity model and Resistance and Capacitance (RC) noise model. The equivalent short circuit resistance, capacitance, and transient current are modeled as nonlinear functions of supply voltage. During simulation, the proposed model can be applied adaptively, that is, in a step-wise manner for each small voltage range. To further validate the performance of our model, we perform sensitivity analysis on the proposed model. With a simple yet accurate power load model, the effect of short circuit current can be investigated, and potentially, system power consumption with billions of transistors can be optimized efficiently.

The remainder of the paper is organized as follows. The problem of power load modeling is formulated in Section 2.1. In Section 2.2 and 2.3 we introduce the proposed RI activity model and RC noise model, followed by sensitivity analysis of our model in Section 2.5. SPICE simulation for empirical value of the equivalent short circuit resistance, capacitance, and transient current is discussed in Section 3. Performance comparison between the proposed method and the reference is shown in Section 4. Finally, we conclude the paper in Section 5 .

\section{NONLINEAR POWER LOAD MODELING METHOD}

A nonlinear power load modeling method is proposed. Such modeling method is capable of speeding up the simulation by adaptive sensitivity analysis.

\subsection{Problem Formulation}

The target is to capture the current model of the CMOS circuits with noise consideration. In this model, the current is a function of 
the supply voltage:

$$
i=f\left(v_{d d}\right)
$$

and we use the summation of the activity power dissipation and the noise fluctuation to represent the total current drop:

$$
i=a\left(v_{d d}\right)+n\left(v_{d d}\right)
$$

where $a\left(v_{d d}\right)$ denotes the activity current load and $n\left(v_{d d}\right)$ denotes the current fluctuation caused by noise.

\subsection{RI Activity Model}

For the activity current load, our circuit model is shown in Fig. 1a, and our method to build the activity model is displayed in Algorithm 1. In our model as Fig. 1a shown, we assume the cycle time is given. With the given activity current load, Algorithm 1 is able to approximate the voltage-dependent current source $i\left(v_{d d}\right)$ and the resistance $R\left(v_{d d}\right)$. The inputs of Algorithm 1 are the activity current load along with the corresponding supply voltage and a user-defined error tolerance, and the outputs are the parameters of the RI model: current source, resistance, and their corresponding piecewise linear supply voltage. The mechanism of the algorithm is to create a user-defined lookup table. Both the nonlinear function and the error tolerance are determined by the user, and thus, the size of the lookup table is flexible.

The spotlights of the proposed algorithm are the nonlinearity of the power load model and the acceleration of the calculation with the support of adaptive piecewise linear slices. The acceleration is achieved with Taylor expansion at a given point, and the region is determined by a given error tolerance $\epsilon$ along with the specified order $n$ as depicted in Equation 3.

$$
\begin{aligned}
f\left(x_{0}+\Delta x\right) & =f\left(x_{0}\right)+\sum_{k=1}^{\infty} \frac{f^{(k)}\left(x_{0}\right)}{k !}(\Delta x)^{k} \\
& =f\left(x_{0}\right)+\sum_{k=1}^{n} \frac{f^{(k)}\left(x_{0}\right)}{k !}(\Delta x)^{k}+\epsilon \\
\Delta x & \approx\left(\left|\frac{n !}{f^{(n)}\left(x_{0}\right)} \epsilon\right|\right)^{(1 / n)}
\end{aligned}
$$

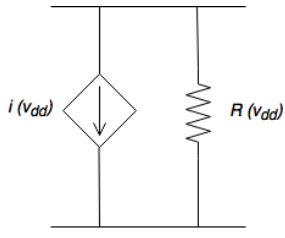

(a) RI activity model

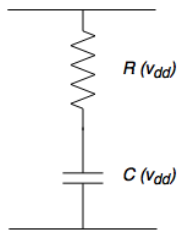

(b) RC noise model
Figure 1: The proposed RI and RC models

\subsection{RC Noise Model}

For the noise analysis, our circuit model is shown in Fig. 1b, and our method to build the noise model is displayed in Algorithm 2. We assume the averaged amplitude and frequency of the noise are provided. To obtain the amplitude and frequency information, phasor analysis is utilized at a pre-processing stage to capture the resistance $R$ and the capacitance $C$ from the noise information.

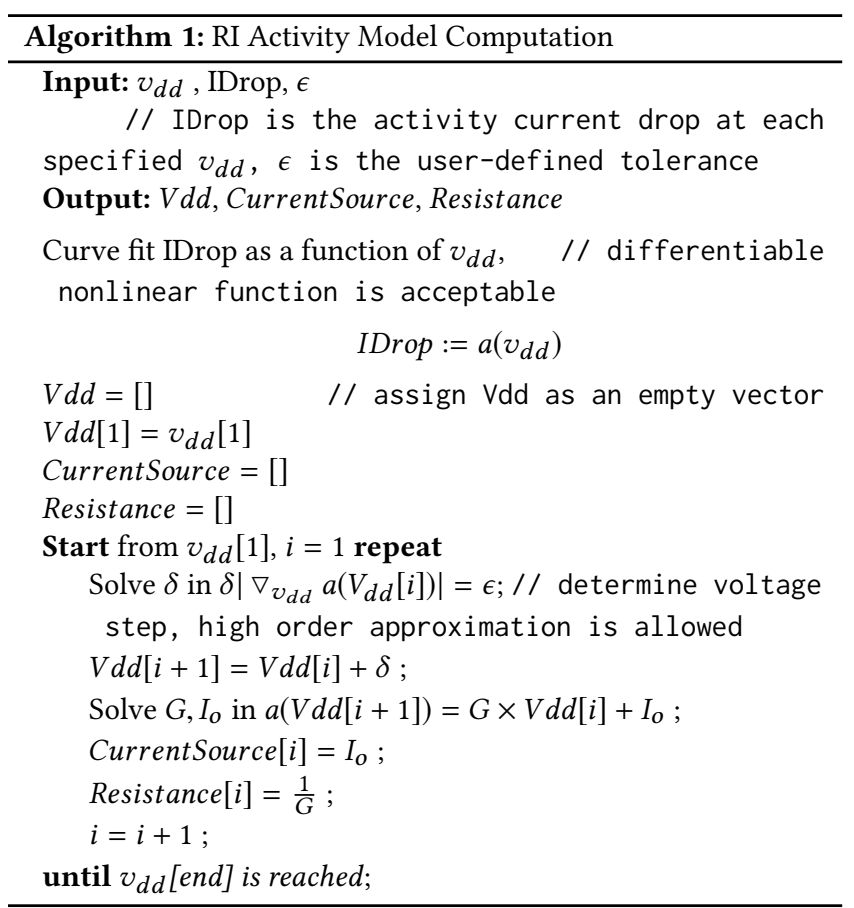

Subsequently, one is able to calculate the adaptive resistance and capacitance at arbitrary supply voltage with Algorithm 2. Algorithm 2 is similar to Algorithm 1 with the difference in the orientations. The inputs of Algorithm 2 are $R, C$ in the noise model, and the outputs are two piecewise linear supply voltages (one for $R$, the other for $C$ ). In other words, two lookup tables are created: one for the resistance, and one for the capacitance. The advantages of $\mathrm{RC}$ noise model are allowing nonlinearity and providing adaptive piecewise linear supply voltages.

\subsection{Nonlinear Power Load Model Simulator}

The circuit diagram of our nonlinear power load model is depicted in Figure 2. We compute the total current under supply voltage fluctuation based on RI and RC models. The simulation of power load circuits is described in Algorithm 3, which adaptively employs the estimated resistance and current source at each simulation step. The strategy of the simulation is to utilize the best corresponding supply voltage (from lookup tables) to complete the calculation at the given time point.

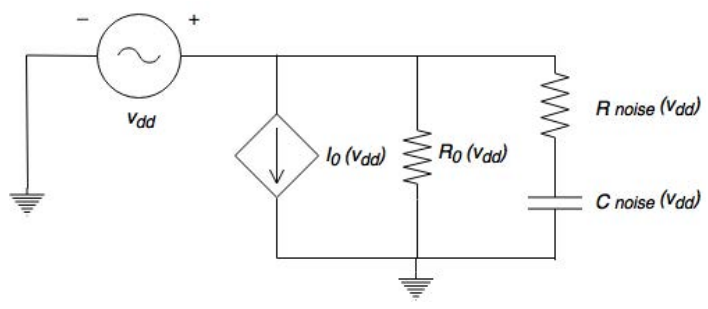

Figure 2: The proposed power load model 

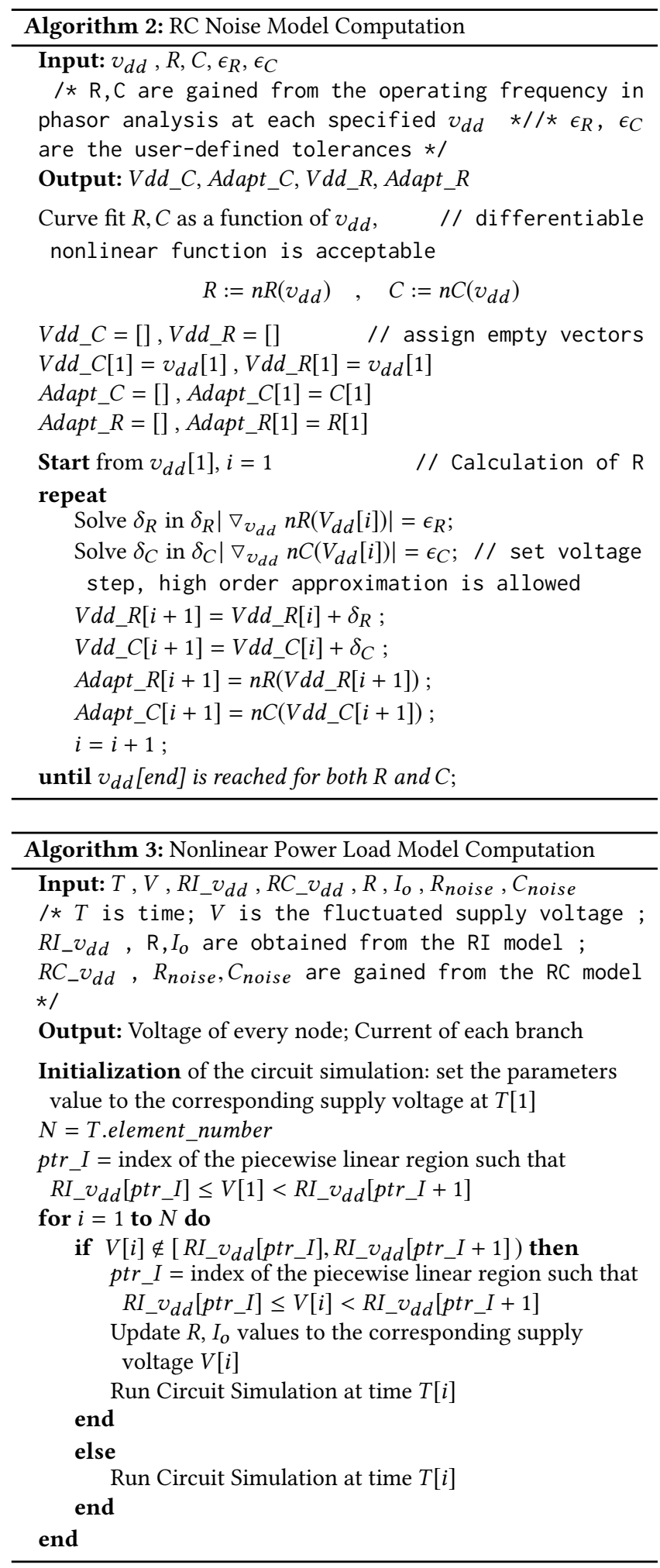

\subsection{Sensitivity Analysis}

Sensitivity equations for the targeted problem can be expressed as Equation 6 [9]. In the sensitivity analysis equations, $i$, the current load, is a variable dependent of time, $v_{d d}$, the supply voltage, represents the parameter of interest, and $i_{o}$ stands for the initial current value. In the second row of Equation $6, \partial_{v_{d d}} i$ and $\partial_{i_{0}} i$ are treated as variables.

$$
D\left[\begin{array}{c}
i \\
\partial_{v_{d d}} i \\
\partial_{i_{0}} i
\end{array}\right]=\left[\begin{array}{c}
f\left(t ; i\left(t ; \boldsymbol{v}_{\boldsymbol{d} \boldsymbol{d}}, i_{0}\right)\right. \\
\partial_{v_{d d}} f\left(t ; i\left(t ; \boldsymbol{v}_{\boldsymbol{d} d}, i_{0}\right), \boldsymbol{v}_{\boldsymbol{d} \boldsymbol{d}}\right)+ \\
\partial_{i} f\left(t ; i\left(t ; \boldsymbol{v}_{\boldsymbol{d} \boldsymbol{d}}, i_{0}\right), \boldsymbol{v}_{\boldsymbol{d} \boldsymbol{d}}\right) \partial_{v_{d d}} i\left(t ; \boldsymbol{v}_{\boldsymbol{d} \boldsymbol{d}}, i_{0}\right) \\
\partial_{i} f\left(t ; i\left(t ; \boldsymbol{v}_{\boldsymbol{d} \boldsymbol{d}}, i_{0}\right), \boldsymbol{v}_{\boldsymbol{d} \boldsymbol{d}}\right) \partial_{i_{0}} i\left(t ; \boldsymbol{v}_{\boldsymbol{d} \boldsymbol{d}}, i_{0}\right)
\end{array}\right]
$$

with the corresponding initial conditions

$$
D\left[\begin{array}{c}
i\left(t_{0}\right) \\
\partial_{v_{d d}} i\left(t_{0}\right) \\
\partial_{i_{0}} i\left(t_{0}\right)
\end{array}\right]=\left[\begin{array}{c}
i_{0} \\
\mathbf{0} \\
\boldsymbol{I}
\end{array}\right]
$$

In our model, the initial conditions are given, so the calculation of the third row in the matrix 6 is not required. From the generalized adjoint network sensitivity calculation [2], the resistive networks' sensitivity is

$$
\frac{-\partial f_{R}}{\partial p_{R}} \phi_{R}(\tau)+\frac{\partial g_{R}}{\partial p_{R}} \psi_{R}(\tau)
$$

In this research, the interest is on the sensitivity of the load current. Therefore, given the fact that the load current is dependent of the supply voltage, the sensitivity equation in interest is the term $\frac{\partial g_{R}}{\partial p_{R}} \phi_{R}(\tau)$. By definition of the adjoint network, all the adjoint branches contribute to the current and voltage change, and the expression for the current load sensitivity becomes

$$
\begin{aligned}
\frac{d}{d t} \frac{d i}{d v_{d d}} & =\frac{d}{d t}\left(\frac{d a\left(v_{d d}\right)}{d v_{d d}}+\frac{d n\left(v_{d d}\right)}{d v_{d d}}\right) \\
& =\frac{d}{d t}\left(\frac{d I\left(v_{d d}\right)}{d v_{d d}}+\left(\frac{d G\left(v_{d d}\right)}{d v_{d d}} v_{d d}+G\left(v_{d d}\right)\right)+\frac{d I \_n o i s e\left(v_{d d}\right)}{d v_{d d}}\right)
\end{aligned}
$$

\section{EXPERIMENTAL RESULTS}

The test cases in this paper were an inverter chain formed of 12 inverters as shown in Fig. 3. In our calculation of the total current, we emphasized on the internal state of the circuit behavior. Therefore, the currents in the first stage (inv 1) and the last stage (inv 12), which were contaminated by the proximity to the input pulse and the output loading capacitor respectively, were removed. The inverter chain simulations were carried out with HSPICE [3].

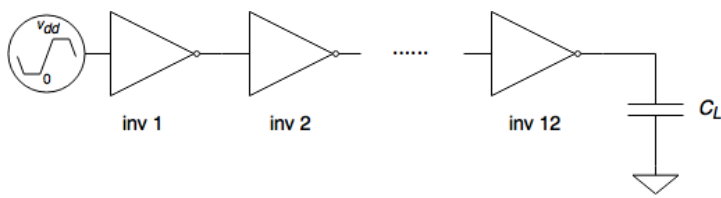

Figure 3: Inverter Chain

To construct the RI Activity model, RC Noise model, and the metrics of the supply voltage fluctuation, we generated the simulation data with HSPICE. In our simulations, two different models from 
ASU predictive model were used: $16 \mathrm{~nm}$ High Performance model and $16 \mathrm{~nm}$ Low Power model. For RI model, the activity current in our experiments was calculated by the averaged current in each pulse cycle ( $8 n s$ per cycle, $1 n s$ for each transition stage, $3 n s$ for each stable state) specified in transient analysis of the inverter chain. For RC model, a sinusoidal wave $\left(100 \mathrm{GHz}, 0.05 * v_{d d} * \sin (2 \pi f t)\right)$ was utilized as the noise input to simulate the fluctuating supply voltage.

The results of the low power and high performance RI, RC models are shown in Fig. 4 and Fig. 5 respectively. Considering RI model, Fig. 4a and Fig. 5a display the global view of the results of the inverter chain's activity current load associated with different supply voltages. Both Fig. 4a and Fig. 5a illustrate the advantages of the proposed RI Activity Model Computation. The X axis is the supply voltage; the $\mathrm{Y}$ axis is the corresponding activity current load. From the figure, we can see that big leaps can be made at the inclined slope, whereas dense piecewise linear regions are observed at the sharp slope. Such adaptive piecewise linear steps not only embrace the accuracy but also reduce the computation time. We observe that larger supply voltage induces larger activity current load, and a nonlinear curve should be employed to fit the curve. For both models, the nonlinear fitting curves in the activity model are polynomials of second order fitting piece-wisely and the tolerances are empirically determined.

For RC model, the performances are demonstrated in Fig. 4b and $5 \mathrm{~b}$. For both figures, the top subplot shows the relation between the supply voltage and the resistance, while the bottom subplot displays the association between the supply voltage and the capacitance. For the low power model, as the supply voltage increases from $0.7 \mathrm{~V}$ to $1.3 \mathrm{~V}$, the resistance first rises and then converges to a certain value around $1.5 \mathrm{k} \Omega$, while the capacitance keeps incrementing. For the high performance model, as the supply voltage increases from $0.5 \mathrm{~V}$ to $0.95 \mathrm{~V}$, the resistance first decreases and then converges to a certain value around $1.2 k \Omega$, while the capacitance keeps incrementing. For both models, the nonlinear fitting curves, the resistance and capacitance in the noise model, are polynomials of second order fitting piece-wisely and the tolerances are empirically determined.

\section{PERFORMANCE EVALUATION}

The inverter chain with fluctuating supply voltage simulation results by HSPICE are the metrics to compare with. Our model generates the results within $3 \%$ error in both high performance and low power models as shown in Tab.1, Tab. 2 and Fig. 6a, 6b. The calculation of the error follows Equation 10. In both tables, the numbers represent the relative error rate between the inverter chain current load value and the current load value of our simulation. The equation applied in this research is Eq. 10. In Tab. 1 and Tab. 2, each row refers to the supply voltage, and each column defines the averaged transient cycle number from the activity current load. Besides the less than $3 \%$ relative error rates, the trends of the current load of our simulation results are consistent with HSPICE in Fig. 6a, 6b. For both high performance and low power models, the current load nonlinearly increases with the increment of the supply voltage.

$$
\text { Error Rate }=\frac{I_{M O D E L}-I_{H S P I C E}}{I_{H S P I C E}} \times 100 \%
$$

\begin{tabular}{|c|ccccc|}
\hline $\begin{array}{c}\text { Low } \\
\text { Power }\end{array}$ & $\begin{array}{c}1 \\
\text { cycle }\end{array}$ & $\begin{array}{c}2 \\
\text { cycles }\end{array}$ & $\begin{array}{c}3 \\
\text { cycles }\end{array}$ & $\begin{array}{c}4 \\
\text { cycles }\end{array}$ & $\begin{array}{c}5 \\
\text { cycles }\end{array}$ \\
\hline $0.75 \mathrm{~V}$ & -1.56 & -1.75 & -1.81 & -1.85 & -1.87 \\
\hline $0.80 \mathrm{~V}$ & -1.69 & -1.96 & -2.05 & -2.09 & -2.12 \\
\hline $0.85 \mathrm{~V}$ & 1.42 & 1.07 & 0.95 & 0.90 & 0.86 \\
\hline $0.90 \mathrm{~V}$ & -1.77 & -2.14 & -2.27 & -2.33 & -2.37 \\
\hline $0.95 \mathrm{~V}$ & 0.52 & 0.13 & -0.01 & -0.07 & -0.11 \\
\hline $1.00 \mathrm{~V}$ & -1.54 & -1.94 & -2.07 & -2.14 & -2.65 \\
\hline $1.05 \mathrm{~V}$ & -2.18 & -2.59 & -2.73 & -2.80 & -2.84 \\
\hline $1.10 \mathrm{~V}$ & -0.96 & -1.40 & -1.54 & -1.61 & -1.66 \\
\hline
\end{tabular}

Table 1: Low Power Model: Error in Percentage (\%)

\begin{tabular}{|c|ccccc|}
\hline $\begin{array}{c}\text { High } \\
\text { Performance }\end{array}$ & $\begin{array}{c}1 \\
\text { cycle }\end{array}$ & $\begin{array}{c}2 \\
\text { cycles }\end{array}$ & $\begin{array}{c}3 \\
\text { cycles }\end{array}$ & $\begin{array}{c}4 \\
\text { cycles }\end{array}$ & $\begin{array}{c}5 \\
\text { cycles }\end{array}$ \\
\hline $0.60 \mathrm{~V}$ & 0.17 & -0.10 & -0.19 & -0.24 & -0.26 \\
\hline $0.65 \mathrm{~V}$ & 1.49 & 1.27 & 1.19 & 1.16 & 1.13 \\
\hline $0.70 \mathrm{~V}$ & -2.03 & -2.20 & -2.25 & -2.28 & -2.29 \\
\hline $0.75 \mathrm{~V}$ & -2.42 & -2.54 & -2.58 & -2.60 & -2.61 \\
\hline $0.80 \mathrm{~V}$ & -2.38 & -2.46 & -2.49 & -2.50 & -2.51 \\
\hline $0.85 \mathrm{~V}$ & -1.63 & -1.69 & -1.71 & -1.72 & -1.72 \\
\hline $0.90 \mathrm{~V}$ & -1.74 & -1.78 & -1.79 & -1.80 & -1.80 \\
\hline
\end{tabular}

Table 2: High Performance Model: Error in Percentage (\%)

Referring to sensitivity analysis, we use low power model as demonstration. The sensitivity analysis $\left(\frac{d i}{d v_{d d}}\right)$ for the total current is exhibited in Fig. 7a and 7b. In Fig. 7a, the X axis is the supply voltage, and $\mathrm{Y}$ axis is the derivative of each parameter of interest. From top to bottom of each subplot, we have $\frac{d I}{d v_{d d}}=\frac{d I_{o}}{d v_{d d}}+\frac{d\left(G v_{d d}\right)}{d v_{d d}}$, $\frac{d R}{d v_{d d}}$, and $\frac{d C}{d v_{d d}}$. For the activity current load, the sensitivity enlarges with the increase of $v_{d d}$. For the resistance in noise model, the sensitivity converges to zero with the increase of $v_{d d}$; regarding the capacitance, the sensitivity does not show the trend of convergence. These results are consistent with the results in Fig. 4b. As for Fig. $7 \mathrm{~b}$, the $\mathrm{X}$ axis is time, and the $\mathrm{Y}$ axis is $\frac{d I}{d v_{d d}}$. Since the noise fluctuates periodically, and given the fact that $R, C$ are determined at initial stage, it is reasonable that the sensitivity of the current load changes periodically.

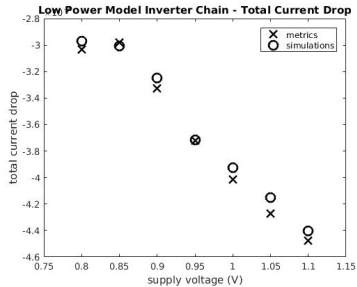

(a) Current Load under Fluctuated Supply Voltage Simulation (low power model)

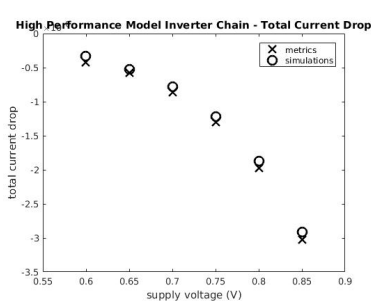

(b) Current Load under Fluctuated Supply Voltage Simulation (high performance model)

\section{Figure 6: Results of the proposed power load model}




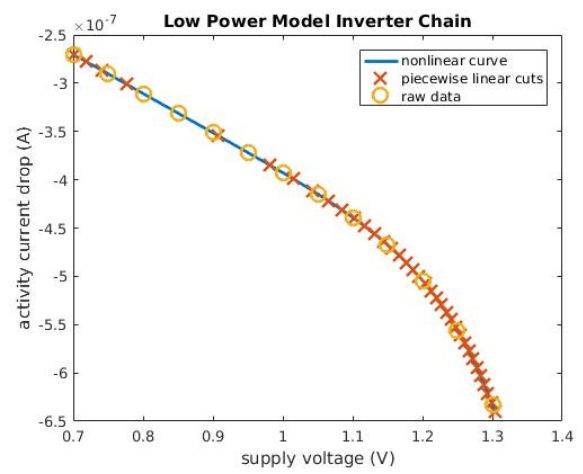

(a) RI model : demonstration of adaptive steps
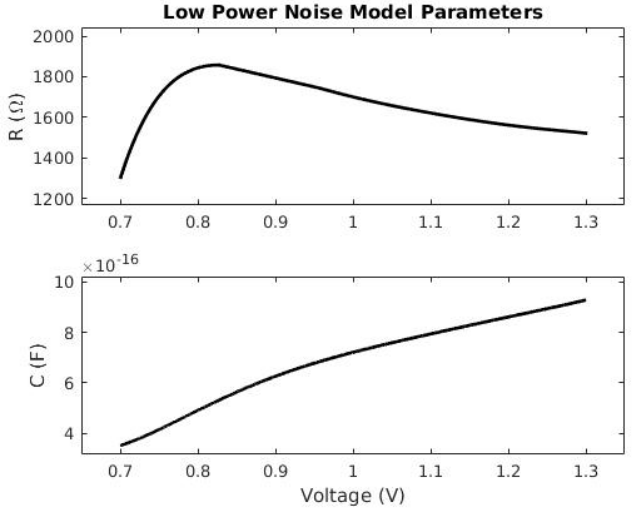

(b) Nonlinear Curves of RI and RC models

Figure 4: Results of Low Power model simulation

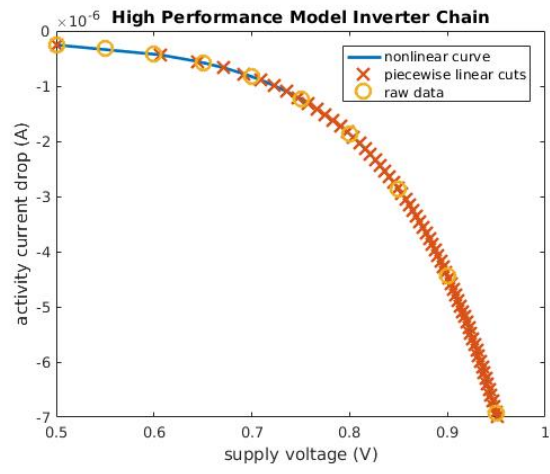

(a) RI model : demonstration of adaptive steps
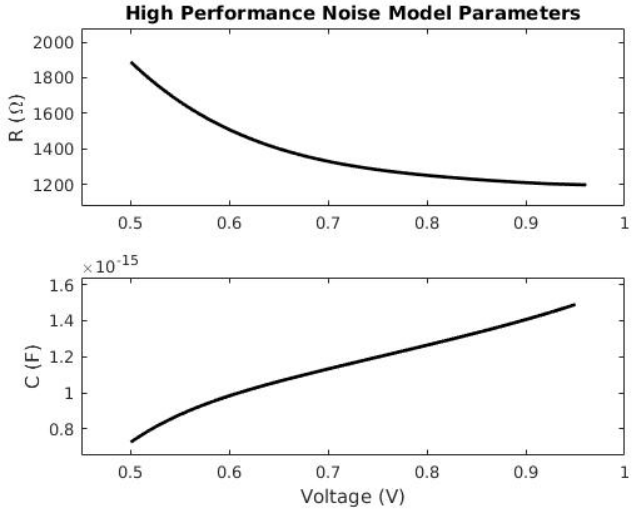

(b) Nonlinear Curves of RC models

Figure 5: Results of High Performance model simulation

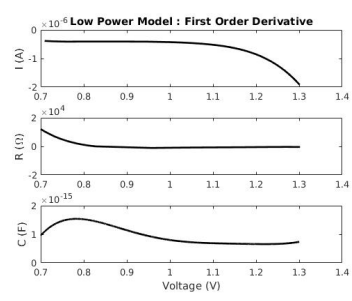

(a) Parameters used in sensitivity analysis (Equation 6) (low power model)

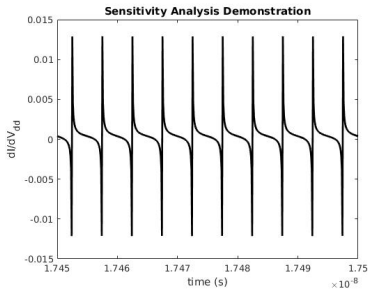

(b) Sensitivity of the total Current Load

Figure 7: Results of sensitivity analysis

\section{CONCLUSIONS}

A nonlinear power load modeling method as a function of the supply voltage is proposed in this paper. To speed up the calculation,

the algorithms to adaptively slice piecewise linear regions are also provided. Moreover, sensitivity analysis methods are given and relying to the sensitivity equations, any parameter of interest can be investigated. In the inverter chain test cases, we reach the performance within $3 \%$ of error in the operating regions of both low power and high performance models. The future work suggested are a more generalized noise model instead of sinusoidal waves, thermal analysis of the current load, and the application of the proposed model to the investigation of the subthreshold regions.

\section{REFERENCES}

[1] Gary Charles and Paul D Franzon. 2012. Comparison of TSV-based PDN-design effects using various stacking topology methods. In Electrical Performance of Electronic Packaging and Systems (EPEPS), 2012 IEEE 21st Conference on. IEEE, 83-86.

[2] Stephen Director and Ronald Rohrer. 1969. The generalized adjoint network and network sensitivities. IEEE Transactions on Circuit Theory 16, 3 (1969), 318-323.

[3] HSPICE. 2017. (2017). https://www.synopsys.com/verification/ams-verification/ circuit-simulation/hspice.htm

[4] ITRS. 2012. (2012). http://www.itrs.net. 
[5] Jingook Kim, Liang Li, Songping Wu, Hanfeng Wang, Yuzo Takita, Hayato Takeuchi, Kenji Araki, Jun Fan, and James L Drewniak. 2012. Closed-form expressions for the maximum transient noise voltage caused by an IC switching current on a power distribution network. IEEE Transactions on Electromagnetic Compatibility 54, 5 (2012), 1112-1124.

[6] Nam Sung Kim, Todd Austin, David Baauw, Trevor Mudge, Krisztián Flautner Jie S Hu, Mary Jane Irwin, Mahmut Kandemir, and Vijaykrishnan Narayanan. 2003. Leakage current: Moore's law meets static power. computer 36, 12 (2003), 68-75.

[7] Woopoung Kim. 2011. Estimation of simultaneous switching noise from frequency-domain impedance response of resonant power distribution networks. IEEE Transactions on Components, Packaging and Manufacturing Technology 1, 9 (2011), 1359-1367.

[8] Istvan Novak. 2008. Power distribution network design methodologies. Intl. Engineering Consortiu.

[9] James Ramsay and Giles Hooker. 2017. Dynamic Data Analysis. (2017).

[10] Larry Smith, Shishuang Sun, Peter Boyle, and Bozidar Krsnik. 2009. System power distribution network theory and performance with various noise current stimuli including impacts on chip level timing. In Custom Integrated Circuits Conference, 2009. CICC'09. IEEE. IEEE, 621-628.

[11] Yosuke Tanaka, Hiroki Takatani, Haruya Fujita, Yoshiaki Oizono, Yoshitaka Nabeshima, Toshio Sudo, Atsushi Sakai, Shiro Uchiyama, and Hiroaki Ikeda 2012. Measurement of sso noise and pdn impedance of $3 \mathrm{~d}$ sip with $4 \mathrm{k}$-io widebus structure. In Electrical Performance of Electronic Packaging and Systems (EPEPS), 2012 IEEE 21st Conference on. IEEE, 91-94.

[12] David C Zhang, Madhavan Swaminathan, and Suzanne Huh. 2012. New power delivery scheme for 3D ICs to minimize simultaneous switching noise for high speed I/Os. In Electrical Performance of Electronic Packaging and Systems (EPEPS), 2012 IEEE 21st Conference on. IEEE, 87-90.

[13] Xiang Zhang, Yang Liu, Ryan Coutts, and Chung-Kuan Cheng. 2013. Power distribution network design optimization with on-die voltage-dependent leakage path. In Electrical Performance of Electronic Packaging and Systems (EPEPS), 2013 IEEE 22nd Conference on. IEEE, 87-90. 